\title{
Synthetic cannabinoid ajulemic acid exerts potent antifibrotic effects in experimental models of systemic sclerosis
}

\author{
Estrella Garcia Gonzalez, ${ }^{1}$ Enrico Selvi, ${ }^{1}$ Epifania Balistreri, ${ }^{1}$ Alfiya Akhmetshina, ${ }^{2}$ \\ Katrin Palumbo, ${ }^{2}$ Sauro Lorenzini, ${ }^{1}$ Pietro Enea Lazzerini, ${ }^{3}$ Cinzia Montilli, ${ }^{3}$ \\ Pier Leopoldo Capecchi, ${ }^{3}$ Monica Lucattelli, ${ }^{4}$ Caterina Baldi, ${ }^{1}$ Elena Gianchechi, ${ }^{3}$ \\ Mauro Galeazzi, ${ }^{1}$ Franco Laghi Pasini, ${ }^{3}$ Jörg H W Distler ${ }^{2}$
}

\begin{abstract}
- Additional material is published online only. To view these files please visit the journal online (http://ard.bmj.com/ content/early/recent)

${ }^{1}$ Department of Clinical Medicine and Immunological Sciences, Rheumatology Unit, Siena, Italy

2Department of Internal Medicine III, University of Erlangen, Erlangen, Germany ${ }^{3}$ Department of Clinical Medicine and Immunological Sciences, Immunology Unit, Siena, Italy

${ }^{4}$ Department of Physiopathology, Experimental Medicine and Public Health, Siena, Italy
\end{abstract}

\section{Correspondence to}

Estrella Garcia Gonzalez, Rheumatology Unit, Department of Clinical Medicine and Immunological Sciences, Siena 53100, Italy: estre79@gmail.com

Accepted 26 February 2012 Published Online First 6 July 2012

\begin{abstract}
Background Cannabinoids modulate fibrogenesis in scleroderma. Ajulemic acid (AjA) is a non-psychoactive synthetic analogue of tetrahydrocannabinol that can bind the peroxisome proliferator-activated receptor- $\gamma$ (PPAR- $\gamma$ ). Recent evidence suggests a key role for PPAR- $\gamma$ in fibrogenesis.

Objective To determine whether AjA can modulate fibrogenesis in murine models of scleroderma.

Material and methods Bleomycin-induced experimental fibrosis was used to assess the antifibrotic effects of AjA in vivo. In addition, the efficacy of AjA in pre-established fibrosis was analysed in a modified model of bleomycin-induced dermal fibrosis and in mice overexpressing a constitutively active transforming growth factor $\beta$ (TGF $\beta$ ) receptor I. Skin fibrosis was evaluated by quantification of skin thickness and hydroxyproline content. As a marker of fibroblast activation, $\alpha$-smooth muscle actin was examined. To study the direct effect of AjA in collagen neosynthesis, skin fibroblasts from patients with scleroderma were treated with increasing concentrations of AjA. Protein expression of PPAR- $\gamma$, and its endogenous ligand 15d-PGJ2, and TGF $\beta$ were assessed before and after AjA treatment.

Results AjA significantly prevented experimental bleomycin-induced dermal fibrosis and modestly reduced its progression when started 3 weeks into the disease. AjA strongly reduced collagen neosynthesis by scleroderma fibroblasts in vitro, an action which was reversed completely by co-treatment with a selective PPAR- $\gamma$ antagonist.
\end{abstract}

Conclusions AjA prevents progression of fibrosis in vivo and inhibits fibrogenesis in vitro by stimulating PPAR- $\gamma$ signalling. Since therapeutic doses of AjA are well tolerated in humans, it is suggested that AjA as an interesting molecule targeting fibrosis in patients with scleroderma.

\section{INTRODUCTION}

Cannabinoids play key roles in several biological processes, including inflammation, immunomodulation and vasomotor responses. ${ }^{12}$ Moreover, the cannabinoid system might also be implicated in the pathogenesis of fibrosis. ${ }^{3} 4$ The endocannabinoid system comprises the two specific cannabinoid receptors, $\mathrm{CB} 1$ and $\mathrm{CB} 2$, their endogenous ligands and the machinery dedicated to endocannabinoid synthesis and degradation. ${ }^{5}$ In experimental models of dermal fibrosis, the CB1 and CB2 cannabinoid receptors modulate fibrogenesis by abrogating the underlying inflammation. ${ }^{67}$ In addition, cannabinoid agonists limit extracellular matrix (ECM) production by disrupting the transforming growth factor $\beta$ (TGF $\beta$ ) cascade, downregulating proliferation and activation of dermal fibroblasts. ${ }^{8} 9$ These data argue for a role of cannabinoids in limiting fibrosis, in addition to their anti-inflammatory and immunomodulatory effects. However, the precise molecular mechanisms remain to be elucidated.

Cannabinoids are not accepted widely as therapeutic agents owing to their psychoactive effects. Therefore, there is growing interest in development of synthetic compounds without cannabimimetic activity on the central nervous system. Ajulemic acid (AjA; 1',1'-dimethylheptyl-tetrahydrocannabinol11-oic acid) is a synthetic analogue of tetrahydrocannabinol, devoid of relevant psychotropic effects. ${ }^{10}$ It is a potent anti-inflammatory and analgesic agent in vivo. ${ }^{10}$ First data in humans indicate that $\mathrm{Aj} A$ is well tolerated and can reduce chronic neuropathic pain without significant psychotropic effects in doses up to $80 \mathrm{mg} /$ day. ${ }^{11}$ Although, AjA may be able to activate CB1-mediated central effects by crossing the blood-brain barrier in mice, ${ }^{12}$ the amount crossed seems not to be sufficient to trigger psychoactivity in humans. ${ }^{13}$ In addition to its affinity for CB1 and $\mathrm{CB} 2$ receptors, cannabinoids, including AjA, are an emerging new class of molecules that can bind to, and activate, the peroxisome proliferatedactivated receptor- $\gamma$ (PPAR- $\gamma$ ). ${ }^{14-17}$ PPAR- $\gamma$ can be activated by some endocannabinoids and, in turn, PPAR- $\gamma$ activation can modulate the endocannabinoid system, suggesting a reciprocal relationship. ${ }^{18}$ Indeed, the agonist effects of endocannabinoids on PPAR- $\gamma$ may contribute to the regulatory effects of endocannabinoids on inflammation, and vasoactivity. ${ }^{10}$ Moreover, recent evidence suggests a key role for PPAR- $\gamma$ in fibrogenesis. Suppression of PPAR- $\gamma$ may contribute to the uncontrolled activation of fibroblasts. ${ }^{19}$ In fact, PPAR- $\gamma$ and its natural occurring ligand 15 -deoxy- $\Delta 12,14$-prostaglandin J2 (15d-PGJ2), exert antifibrotic effects by suppressing collagen production and fibroblast activation in bleomycin-induced fibrosis. ${ }^{20} 21$ 
Since systemic sclerosis (SSc) is an autoimmune disease in which vascular injury and inflammation lead to progressive fibrosis of tissues, ${ }^{22}$ the cannabinoid system and PPAR- $\gamma$ have been suggested as potential therapeutic targets. Considering that therapeutic doses of AjA simultaneously activate PPAR- $\gamma$ and the cannabinoid system without affecting the central nervous system, we hypothesised that $\mathrm{AjA}$ might also be effective in preventing fibrosis in experimental models of SSc.

\section{MATERIAL AND METHODS}

\section{Animal models of experimental dermal fibrosis}

To asses in vivo the potential role of AjA as an antifibrotic agent, three different animal models were analysed. AjA was evaluated in bleomycin-induced dermal fibrosis, characterised primarily by inflammation, which typically occurs in the early phases of SSc. Skin fibrosis was induced in two groups of 6-week-old DBA/2J mice by local injection of bleomycin. Animals were treated with bleomycin alone (BLM group; n=8), bleomycin plus AjA 1 mg/kg/ day (BLM/AjA group; $\mathrm{n}=8$ ) and $0.9 \% \mathrm{NaCl}$ saline solution (control group; $\mathrm{n}=8$ ). Briefly, $100 \mu \mathrm{l}$ of BLM dissolved in $0.9 \% \mathrm{NaCl}$ at a concentration of $0.5 \mathrm{mg} / \mathrm{ml}$ was administered every other day, in well-defined areas $\left(1 \mathrm{~cm}^{2}\right)$ of the upper back. ${ }^{23}$ One group of BLM-challenged mice received orally AjA (JB Therapeutics, Newton, Massachusetts, USA) $1 \mathrm{mg} / \mathrm{kg} /$ day, dissolved in seed oil. Subcutaneous injections of $100 \mu \mathrm{l} 0.9 \% \mathrm{NaCl}$ were used as controls. After 21 days, animals were killed by cervical dislocation. The injected skin was removed and processed for analysis (see online supplementary text).

To test AjA on established fibrosis less dependent on inflammatory mechanisms, a modified model of bleomycin-induced dermal fibrosis was assessed, ${ }^{24}$ in which mice were challenged with bleomycin for 6 weeks and AjA treatment started during the last 3 weeks of bleomycin injections. Briefly, one group of mice was killed after receiving subcutaneous bleomycin injections for 6 weeks. The second group received bleomycin injections for 3 weeks followed by $0.9 \% \mathrm{NaCl}$ injections for the next 3 weeks, to control for spontaneous regression of fibrosis. The third group was challenged with bleomycin for 6 weeks and was treated in parallel with $\mathrm{AjA}$ (1 $\mathrm{mg} / \mathrm{kg} /$ day; oral administration) during the final 3 weeks of bleomycin injections. Two groups of mice, receiving subcutaneous injections of $100 \mu \mathrm{l} 0.9 \% \mathrm{NaCl}$ for 3 weeks and 6 weeks, respectively, were used as controls. The injected skin was removed and processed for analysis (see online supplementary text).

To assess the effect of AjA in a different model of scleroderma fibrosis, a TGF $\beta$-dependent model of dermal fibrosis was studied. Dermal fibrosis was induced in pathogen-free male C57BL/6 mice by intracutaneous injections of attenuated type $\mathrm{V}$ adenoviruses overexpressing a constitutively active TGF $\beta$ receptor I (AdTGFbRI) as described. ${ }^{25}$ Three groups of mice were analysed. One group was treated in parallel orally with AjA (1 mg/ $\mathrm{kg} /$ day) for the last 4 weeks. Mice infected with attenuated type $\mathrm{V}$ adenoviruses encoding only for LacZ and sham treated with the solvent of AjA served as controls. Animals were killed after 8 weeks by cervical dislocation and back skin was removed for analysis (see online supplementary text).

Each treatment group consisted of eight mice. The local ethical committee approved all animal experiments.

\section{Patients and fibroblast cultures}

Dermal fibroblasts were obtained by a $5 \mathrm{~mm}$ punch biopsy from the leading edge of the affected skin on the forearm of five patients affected by diffuse cutaneous SSc $(\mathrm{dcSSc})$ in the fibrotic
Table 1 Clinical and demographic characteristics of the patients with diffuse cutaneous systemic sclerosis (dcSSc)*

\begin{tabular}{ll}
\hline Characteristics & Value \\
\hline Sex (M/F), $n$ & $1 / 4$ \\
Age, median (range), years & $52(35-72)$ \\
Disease duration, median (range), years ${ }^{\dagger}$ & $6(3-12)$ \\
Diffuse cutaneous disease & 5 \\
Number of Scl-70 positive & 5 \\
Potential disease-modifying agents & 0 \\
Prostacyclin analogues & 5 \\
Calcium channel blockers & 4 \\
Prokinetics & 4 \\
\hline
\end{tabular}

${ }^{*}$ dcSSc was determined according to LeRoy et al criteria. ${ }^{26}$

${ }^{\dagger}$ From the first non-Raynaud's manifestation.

phase. All patients fulfilled the criteria for dcSSc classification proposed by LeRoy et al. ${ }^{26}$ No patient was treated with an immunosuppressive agent or corticosteroids, nor were they taking cannabinoids for either recreational or therapeutic use at the time of biopsy (Table 1). Control fibroblasts were obtained from skin biopsy specimens from five healthy age- and sex-matched volunteers. Skin fibroblasts were expanded by outgrowth culture in Dulbecco's modified Eagle's medium (DMEM; Gibco Invitrogen, Grand Island, NY USA) as described previously. ${ }^{27}$ Fibroblasts from passages 3-6 were used for the experiments. All patients and healthy volunteers provided written informed consent using forms approved by the local institutional review boards.

\section{Stimulation experiments}

dcSSc and healthy dermal fibroblasts were cultured in DMEM containing $2.5 \%$ of fetal calf serum for $24 \mathrm{~h}$ before the experiments. Dermal fibroblasts were incubated with AjA (JB Therapeutics), dissolved in dimethyl sulphoxide at $0.1,1,5$ and $10 \mu \mathrm{M}$ concentrations for $24 \mathrm{~h}$. Further experiments were performed by incubating dermal fibroblasts for 15 min before AjA treatment with the PPAR- $\gamma$ irreversible antagonist GW9662 (Tocris Bioscience, Bristol, UK), at a concentration of 1 and $10 \mu \mathrm{M}$. (see online supplementary text).

\section{Statistical analysis}

Data are expressed as the mean \pm SD. Analysis of variance was used to compare multiple means, followed by the Student-Newman Keuls post hoc test (Sigma-StatV.3.5; Sigma Stat, Ashburn, Virginia, USA). p Values $<0.05$ were considered significant.

\section{RESULTS}

\section{Ajulemic acid prevented bleomycin-induced dermal fibrosis in vivo}

To evaluate the potential of AjA as an antifibrotic agent in vivo, we tested its efficacy in bleomycin-induced dermal fibrosis. Animals were treated with bleomycin alone (BLM group), bleomycin plus AjA $1 \mathrm{mg} / \mathrm{kg} /$ day (BLM/AjA group) and $0.9 \% \mathrm{NaCl}$ saline solution (control group). After treatment, the BLM group and BLM/AjA group did not differ in body weight, and no other signs of toxicity of AjA such as ruffled fur or reduced activity were recorded.

Skin fibrosis was quantified at day 21 by determining dermal thickness and hydroxyproline content of lesional skin. Skin from the BLM group showed dense accumulation of ECM in the dermis and accumulation of inflammatory cells in the deeper dermal layers and perivascular spaces. In addition, the subcutaneous fat tissue was largely replaced by connective tissue. (figure $1 \mathrm{~A}$ ) A twofold increase in dermal thickness was seen in mice injected 

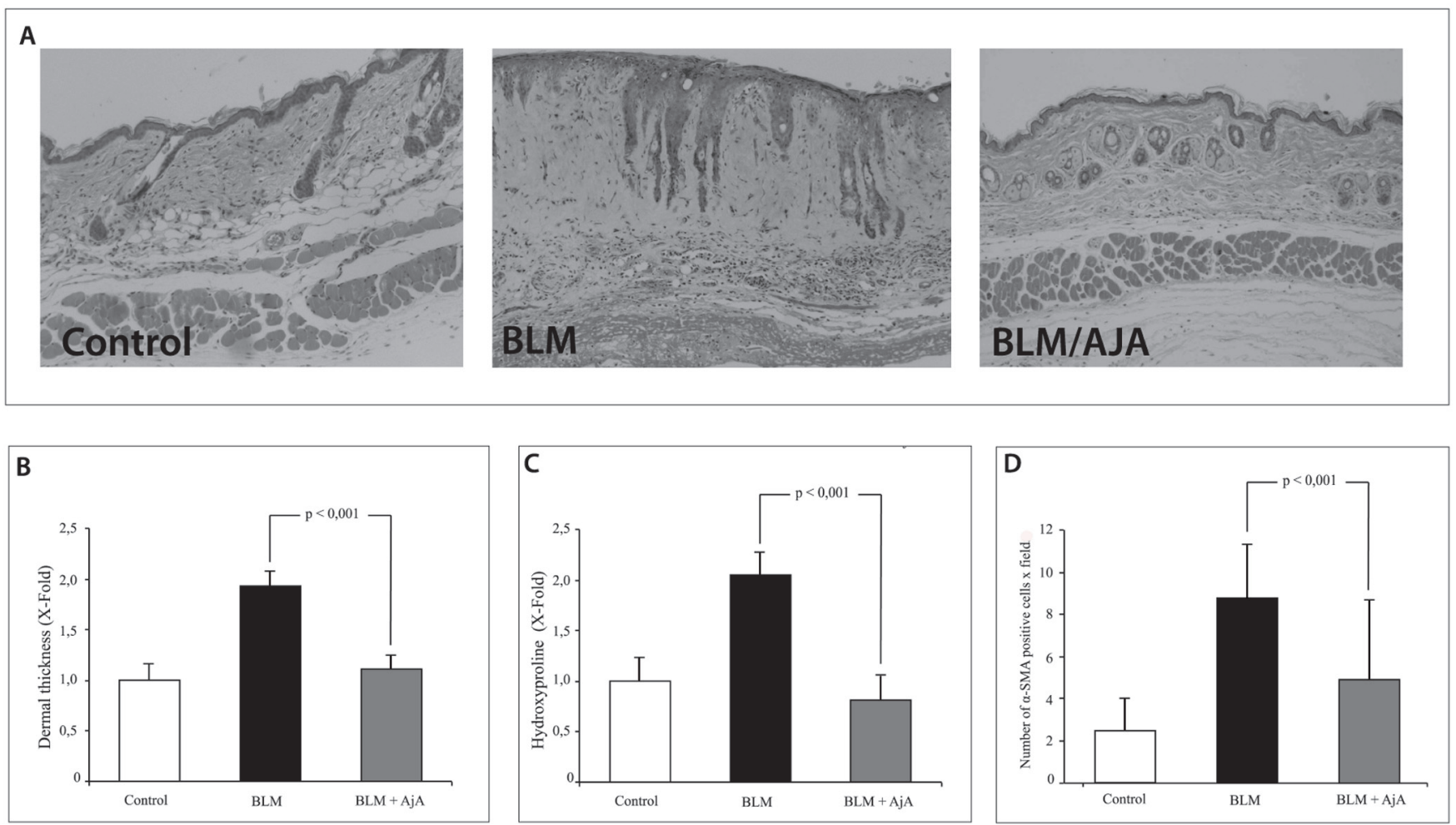

Figure 1 Ajulemic acid (AjA) prevents dermal fibrosis induced by bleomycin (BLM). (A) Dermal thickening, epidermal hypertrophy, subcutaneous fat atrophy and leucocyte infiltration in the deep dermal layers and perivascular spaces were seen in lesional skin from BLM-challenged mice. Treatment with AjA (1 mg/kg/day) preserved dermal architecture and subcutaneous fat layers, and reduced the leucocyte infiltration markedly. Haematoxylin and eosin staining. Original magnification $\times 10$. (B) Histograms showing dermal thickness of lesional skin. After 21 days of treatment with AjA (1 mg/kg/day) dermal thickness was equal to that of the control group. (C) Histograms showing hydroxyproline content of lesional skin. After 21 days of treatment with AjA (1 mg/kg/day) dermal thickness was equal to that of the control group. (D) Histograms showing significant reduction in the number of $\alpha$-smooth muscle actin ( $\alpha$-SMA)-positive spindle-shaped fibroblastic cells per microscopic high field after AjA treatment. Each treatment group, $n=8$. All values are mean \pm SD. Analysis of variance was used to compare multiple means, followed by the Student-Newman Keuls post hoc test for multiple comparisons.

with BLM compared with controls $(\mathrm{p}<0.001)$. Oral administration of AjA (1 mg/kg/day) prevented development of skin fibrosis, and reduced skin thickness nearly to control levels $(\mathrm{p}<0.001$ compared with mock treated mice) (figure 1B). Furthermore, in the BLM/AjA group the subcutaneous leucocyte infiltration, the accumulation of ECM and the fat layer replacement were reduced (figure $1 \mathrm{~A}$ ).

Consistent with the histology, production of collagen, determined by quantification of dermal hydroxyproline content, was reduced substantially in mice treated with $\mathrm{AjA}$ (figure $1 \mathrm{C}$ ). In the BLM group, levels of hydroxyproline were more than twofold greater than in controls $(\mathrm{p}<0.001)$. AjA treatment $(1 \mathrm{mg} / \mathrm{kg} /$ day $)$ completely abrogated the collagen deposition induced by BLM $(\mathrm{p}<0.001)$.

Myofibroblasts, characterised by the cytoskeletal protein $\alpha$-smooth muscle actin, are considered one of the major cellular mediators of fibrosis in SSc. In BLM-challenged mice the number of $\alpha$-smooth muscle actin-positive spindle-shaped fibroblastic cells per high-power field was increased twofold compared with controls $(p<0.001)$. AjA treatment significantly reduced the number of myofibroblasts in lesional skin up to $26 \pm 5 \%$ $($ mean \pm SD) $(p<0.05)$ (figure 1D).

\section{Ajulemic acid reduced progression of fibrosis in vivo}

To assess whether AjA is effective in an in vivo model of dermal fibrosis that is largely independent of autoimmune and inflammatory phenomena, we tested its efficacy in the constitutively active TGF $\beta$ receptor type I mouse model (AdTGFbRI). AdTGFbRI mice are characterised by generalised dermal fibrosis and fibroblast-specific activation of TGF $\beta$ signalling. ${ }^{23}$ After 8 weeks AdTGFbRI mice showed twofold increase in dermal thickness compared with AdLacZ control mice $(p<0.05)$. Oral administration of AjA (1 mg/kg/day) for the last 4 weeks reduced development of skin thickness up to $30 \pm 6 \%($ mean $\pm S D)$ compared with AdTGFbRI mice $(p<0.05)$ (Figure 2A,B). AjA treatment (1 mg/kg/day) induced a mean reduction of $30 \pm 13 \%$ (mean $\pm S D$ ) in hydroxyproline content $(\mathrm{p}<0.05)$ (figure $2 \mathrm{C})$. AjA treatment also significantly reduced the number of myofibroblasts in lesional skin up to $26 \pm 3 \%$ $(\mathrm{p}<0.001)$ (figure 2D).

To further evaluate the efficacy of AjA for the treatment of established fibrosis, we used a 6- week model of bleomycininduced dermal fibrosis. Consistent with previous studies, dermal thickness increased by $76 \pm 4 \%$ (mean $\pm \mathrm{SD}$ ) ( $p<0.05$ vs $\mathrm{NaCl}$ controls) after 6 weeks of treatment with bleomycin. To assess the effects of AjA, mice challenged with bleomycin for 6 weeks were treated in parallel with $\mathrm{AjA}$ (1 mg/kg/day) during the final 3 weeks of bleomycin injections. Dermal thickness in mice treated with AjA for the last 3 weeks was reduced by $11 \pm 17 \%$ (mean \pm SD) compared with 6-week bleomycin-challenged mice $(p<0.05)$. Furthermore, dermal thickness in mice treated with AjA did not differ significantly from mice challenged with bleomycin for 

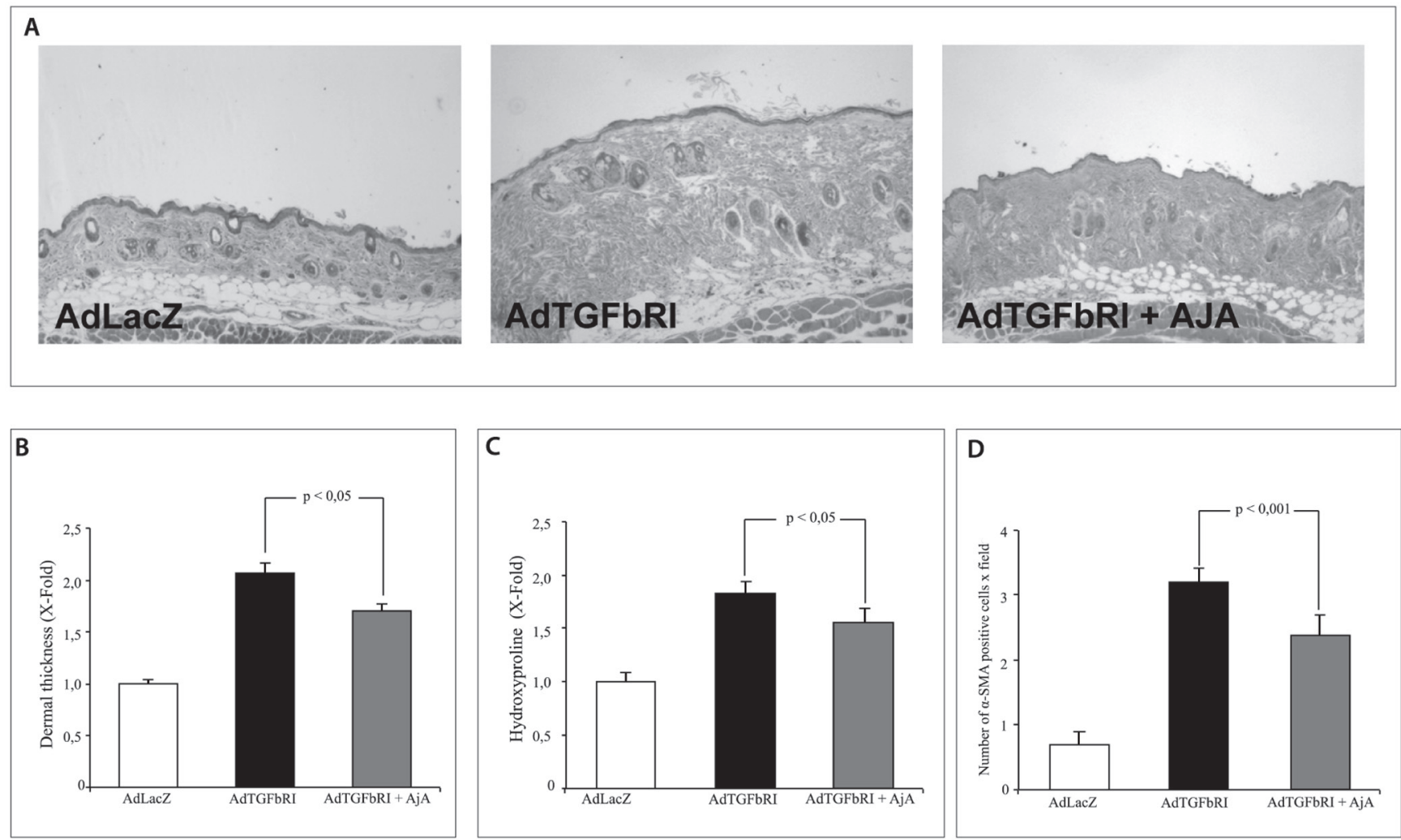

Figure 2 Antifibrotic effects of ajulemic acid (AjA) in AdTGFbRI mice. (A) Dermal fibrosis in AdTGFbRI mice treated with AjA (1 mg/kg/day) was reduced compared with mock-treated mice. Haematoxylin and eosin staining. Original magnification $\times 10$. (B) Histograms showing a significant reduction in dermal thickness of AdTGFbRI lesional skin after AjA treatment. (C) Hydroxyproline content of AdTGFbRI lesional skin was reduced after AjA treatment. (D) Histograms showing significant reduction in $\alpha$-smooth muscle actin ( $\alpha$-SMA)-positive spindle-shaped fibroblastic cells per microscopic high field after AjA treatment of AdTGFbRI mice. Each treatment group, $n=8$. All values are mean $\pm S D$. Analysis of variance was used to compare multiple means, followed by the Student-Newman Keuls post hoc test for multiple comparisons.
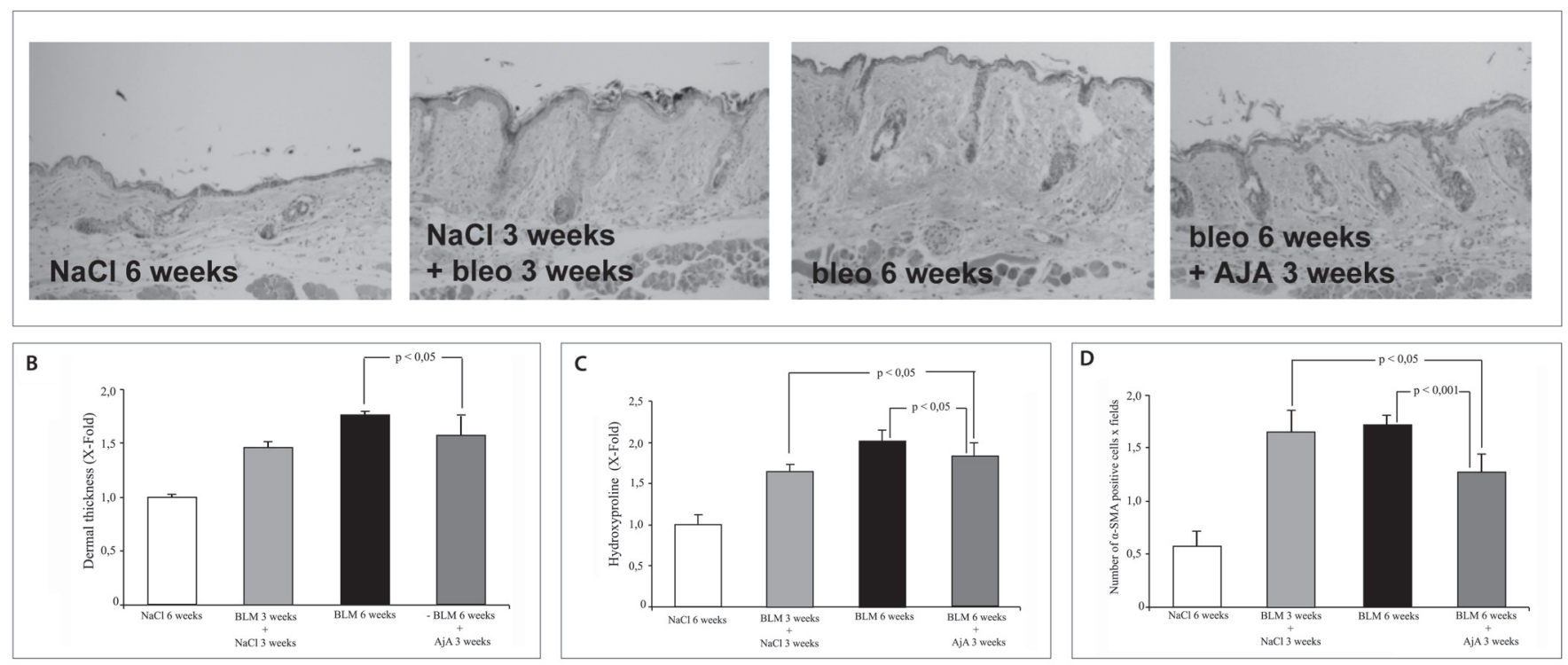

Figure 3 Ajulemic acid $(\mathrm{Aj} A)$ inhibits progression of dermal fibrosis in a modified model of 6 weeks bleomycin (BLM)-induced fibrosis. (A) Treatment with AjA (1 mg/kg/day) prevented further matrix accumulation, but did not induced regression of pre-existing fibrosis. Haematoxylin and eosin staining. Original magnification $\times 10$. (B) Treatment with AjA reduced dermal thickness of lesional skin for the last 3 weeks. (C) Histograms showing hydroxyproline content of lesional skin. AjA treatment disrupted collagen deposition for the last 3 weeks, without regression of pre-existing extracellular matrix accumulation. (D) Histograms showing a significant reduction in the number of $\alpha$-smooth muscle actin ( $\alpha$-SMA)-positive spindle-shaped fibroblastic cells per microscopic high field after treatment with AjA. Each treatment group, $n=8$. All values are mean \pm SD. Analysis of variance was used to compare multiple means, followed by the Student-Newman Keuls post hoc test for multiple comparisons. 
3 weeks (figure 3A,B). Consistently, a twofold increase of levels of hydroxyproline was recorded in the 6-week bleomycin-challenged group compared with controls $(p<0.001)$. AjA treatment for the last 3 weeks reduced collagen deposition by $10 \pm 8 \%($ mean $\pm S D)$ $(p<0.05)$. Thus, AjA administration for the last 3 weeks of bleomycin challenge stopped further progression of fibrosis, but did not alter pre-existing ECM accumulation (figure 3C). The number of myofibroblasts after AjA treatment was significantly reduced in lesional skin up to $25 \pm 16 \%$ (mean \pm SD) compared with bleomycin-challenged mice $(\mathrm{p}<0.05)$ (figure 3D).

\section{Ajulemic acid inhibited collagen neosynthesis in dcSSc fibroblasts through PPAR- $\gamma$}

To investigate whether AjA directly inhibits collagen synthesis in cultured fibroblasts, we quantified the levels of procollagen type I propeptide (PIP) in supernatant from dcSSc and healthy cultured fibroblasts treated with AjA. AjA (0.1 to $10 \mu \mathrm{M})$ reduced the levels of PIP released from dcSSc fibroblasts in a dose-dependent manner. Maximal inhibition was observed at $10 \mu \mathrm{M}$ concentration with a mean PIP reduction up to $60 \pm 7 \%(p<0.001)$ (figure 4). Similar results were seen in healthy dermal fibroblasts (data not shown).

AjA did not exert toxic effects on fibroblasts at the concentrations used. The metabolic activity measured by the MTT assay was not affected by treatment with $\mathrm{AjA}$ in concentrations up to $10 \mu \mathrm{M}$ (data not shown).

To evaluate a PPAR- $\gamma$ pathway dependency, dcSSc and healthy fibroblasts treated with AjA were preincubated with the highly selective PPAR- $\gamma$ antagonist GW9662 (1-10 $\mu \mathrm{M})$. The inhibitory effect of AjA on collagen production was completely prevented by GW9662 at $10 \mu \mathrm{M}$ concentration ( $\mathrm{p}<0.05$ ) (figure $5 \mathrm{~A}$ ).

In addition, we analysed the effect of $\mathrm{Aj} A$ on expression of the endogenous PPAR- $\gamma$ ligand 15d-PGJ2. The levels of 15d-PGJ2 were reduced in supernatants of dcSSc fibroblasts compared with controls $(p<0.001)$. However, incubation with AjA strongly increased the levels of $15 \mathrm{~d}-\mathrm{PGJ} 2$ in a dose-dependent manner $(p<0.001)$ (figure 5B).

Further, we assessed PPAR- $\gamma$ expression in dcSSc and healthy fibroblasts. The protein levels of PPAR $-\gamma$ were reduced in dcSSc fibroblasts compared with healthy controls. However, treatment with AjA (5 $\mu \mathrm{M}$ and $10 \mu \mathrm{M})$ significantly increased the expression of PPAR- $\gamma$ in dcSSc fibroblasts $(p<0.001)$ (figure $5 C$ ).

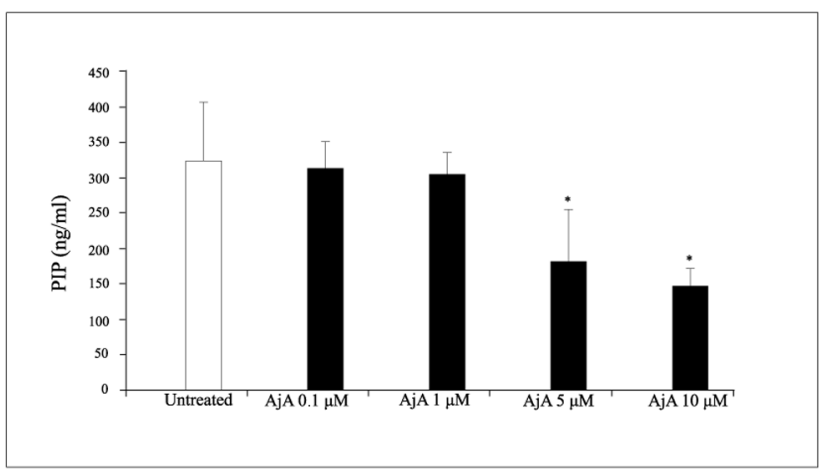

Figure 4 Ajulemic acid $(\mathrm{Aj} A)$ decreases collagen neosynthesis in diffuse cutaneous systemic sclerosis (dcSSc) fibroblasts in a dosedependent manner. Treatment with $\operatorname{AjA}(0.1,1,5$ and $10 \mu \mathrm{M})$ induced a dose-dependent reduction of supernatant procollagen type I propeptide (PIP) levels from SSc fibroblasts. Results are the mean \pm SD of five separate experiments. ${ }^{*} p<0.001$ versus untreated.
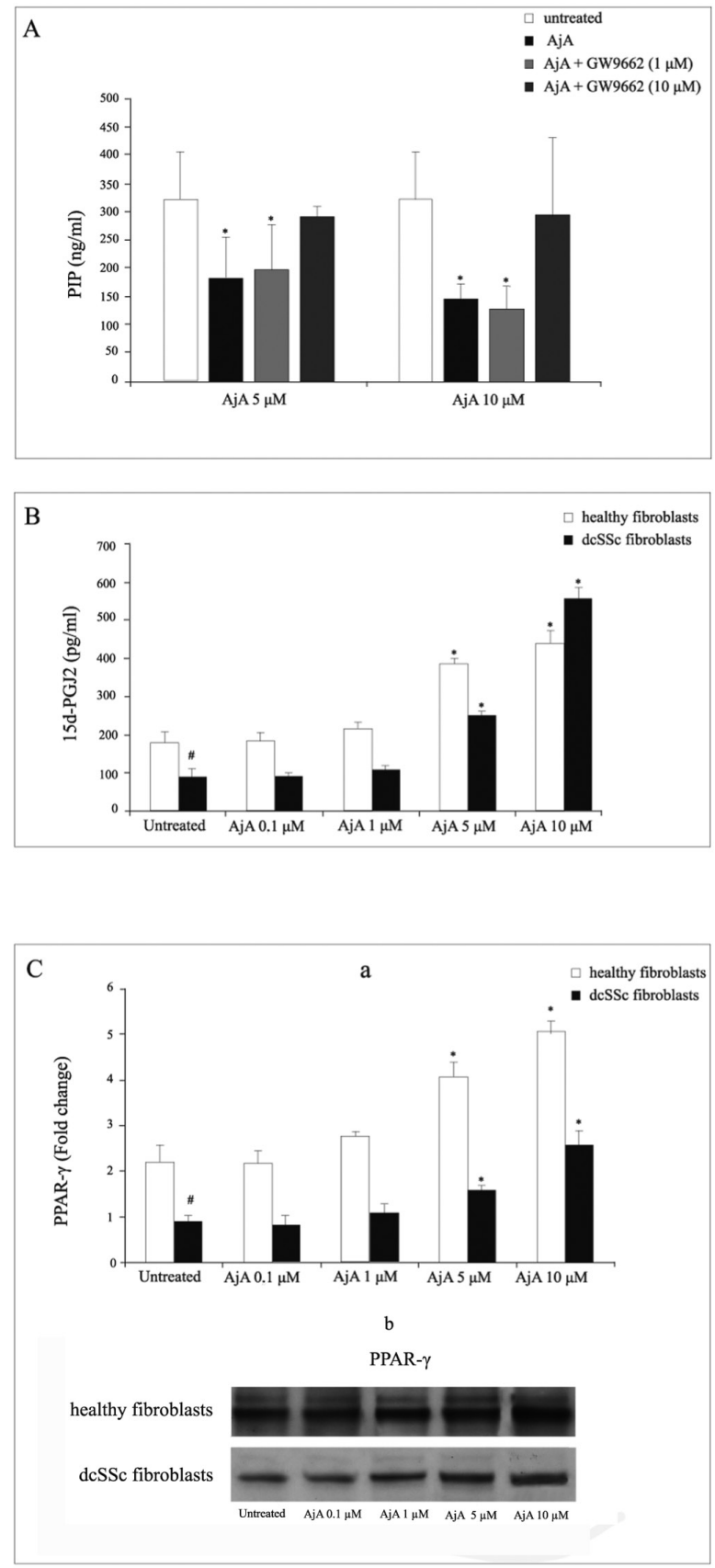

Figure 5 The anti-fibrotic effects of ajulemic acid $(\mathrm{AjA})$ are mediated by peroxisome proliferator-activated receptor- $\gamma$ (PPAR- $\gamma$ ). (A) AjA inhibitory effect on procollagen type I propeptide (PIP) production was completely reversed by preincubation of cells with the PPAR- $\gamma$ irreversible antagonist GW9662 at $10 \mu \mathrm{M}$. Results are the mean \pm SD of five separate experiments. ${ }^{*} p<0,001$ versus untreated. (B) AjA enhanced production of $15 d-P G J 2$ in systemic sclerosis (SSc) fibroblasts. Smaller amounts of the natural ligand of PPAR- $\gamma$, 15d-PGJ2, were found in the supernatant from diffuse cutaneous $\mathrm{SSc}$ (dcSSc) fibroblasts compared with the amounts from healthy controls. After AjA treatment supernatant levels of $15 \mathrm{~d}-\mathrm{PGJ} 2$ increased significantly in a dose-dependent manner. Results are the mean $\pm S D$ of five separate experiments. $\# p<0.001$ versus healthy fibroblasts; ${ }^{*} p<0.001$ vs untreated. (C) AjA increased PPAR- $\gamma$ expression in dcSSc fibroblasts. dcSSc and healthy fibroblasts were treated with $\mathrm{AjA}$ at increasing concentrations $(0.1,1,5$ and $10 \mu \mathrm{M})$. At $24 \mathrm{~h}$ after treatment, cells were lysed and PPAR- $\gamma$ protein levels were analysed by western blot (a) and densitometry (b). Lower PPAR- $\gamma$ levels were seen in dcSSc fibroblasts than in healthy controls. After AjA treatment ( $5 \mu \mathrm{M}$ and $10 \mu \mathrm{M}$ ) PPAR- $\gamma$ protein expression increased significantly. Results are the mean $\pm S D$ of three separate experiments. $\# p<0.001$ versus healthy fibroblasts; ${ }^{*} p<0.001$ versus untreated. 
A

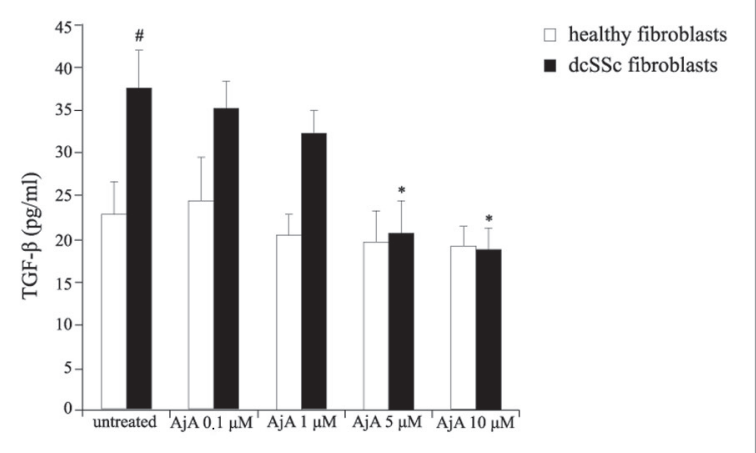

B

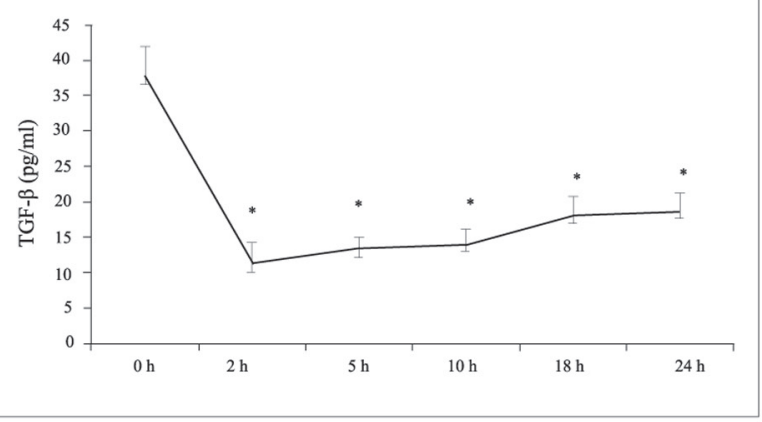

Figure 6 Ajulemic acid $(\mathrm{AjA})$ downregulated the release of transforming growth factor $\beta$ (TGF $\beta$ ) in diffuse cutaneous systemic sclerosis (dcSSc) fibroblasts. (A) AjA (5 and $10 \mu \mathrm{M}$ ) treatment resulted in a significant reduction of supernatant TGF $\beta$. Results are the mean $\pm S D$ of five separate experiments. $\# p<0.001$ versus healthy fibroblasts; * $p<0.001$ versus untreated. (B) Maximum TGF $\beta$ inhibition in dcSSc fibroblasts was seen after $2 \mathrm{~h}$ of AjA treatment $(10 \mu \mathrm{M})$ and remained significantly downregulated for $24 \mathrm{~h}$. Results are the mean $\pm S D$ of five separate experiments. ${ }^{*} p<0.001$ versus untreated.

(TGF) is considered a major profibrotic cytokine in orchestrating the uncontrolled activation of SSc fibroblasts with consequent overproduction of ECM. Moreover, in fibroblasts, TGF $\beta$ signalling is inversely related to PPAR- $\gamma$ physiology function. ${ }^{28}$ Since AjA suppressed collagen production through PPAR- $\gamma$ activation, and in vivo reduced dermal fibrosis in the constitutively active TGF $\beta$ receptor type I mouse model (AdTGFbRI), we evaluated its ability to downregulate TGF $\beta$ in vitro. TGF $\beta$ concentrations in supernatants from dcSSc fibroblasts were significantly higher than TGF $\beta$ concentrations in cultures from healthy controls $(p<0.001)$. Upon incubation with AjA, a significant dose-dependent reduction in TGF $\beta$ concentrations (up to $50 \%$ ) was seen in supernatants from dcSSc fibroblasts $(p<0.001)$, with maximum suppression seen at $2 \mathrm{~h}$ (figure 6).

\section{DISCUSSION}

Results of experiments presented here indicate that AjA efficiently prevents bleomycin-induced dermal fibrosis in mice. In addition, this cannabinoid can inhibit progression of ECM deposition in animal models of established skin fibrosis. In dcSSc fibroblasts, AjA inhibits collagen synthesis through activation of PPAR- $\gamma$.

Modulation of the $\mathrm{CB} 1$ and $\mathrm{CB} 2$ receptors limits skin fibrosis by reducing inflammation. ${ }^{67}$ Although the synthetic tetrahydrocannabinol analogue $\mathrm{Aj} A$ is a weak ligand of the classical cannabinoids receptors $\mathrm{CB} 1$ and $\mathrm{CB} 2,{ }^{10}$ we suggest that AjA may also act to reduce fibrosis directly through a PPAR$\gamma$-mediated mechanism. Consistent with this notion are data which indicate that the high-affinity cannabinoid receptor agonist WIN55, 212-2 exerts antifibrotic effects that are not mediated by the classic cannabinoid receptors. ${ }^{89}$ A close relationship between the endocannabinoid system and PPAR- $\gamma$ signalling has been postulated. ${ }^{18}$ Accumulating evidence suggests that impaired expression and function of PPAR- $\gamma$ is important in the pathogenesis of fibrosis in SSc. ${ }^{28}$ Such diminished activity is counterbalanced by uncontrolled TGF $\beta$ signalling, thereby triggering fibrogenesis. ${ }^{29}$ Cross-talk between these two mediators orchestrates the mechanisms of normal wound healing. ${ }^{20}$ The activation of PPAR- $\gamma$ by its natural ligands controls fibrotic responses. PGJ2 in a physiological condition increases during the resolution phase of wound healing by blocking fibroblast activation and collagen neosynthesis. ${ }^{28}$ Upon chronic fibrogenic stimulation the expression and function of PPAR- $\gamma$ are reduced, leading to unbalanced collagen neosynthesis driven by TGF $\beta$ signalling. Accordingly, PPAR $-\gamma$ activation by PGJ2 results in a robust reduction of inflammatory infiltrates and progression of fibrosis in murine models of bleomycin-induced fibrosis. ${ }^{21}$

Consistent with these findings, we observed that the increased release of TGF $\beta$ is mirrored by a concomitant decrease of PPAR- $\gamma$. We demonstrate that AjA reduces release of TGF $\beta$ from $\mathrm{dcSSc}$ fibroblasts and that AjA can counteract the progression of fibrosis in vivo in an overactivated TGF $\beta$ pathway mouse model. Moreover, $\mathrm{AjA}$ also stimulates expression of the endogenous PPAR- $\gamma$ ligand PGJ2. By inhibiting TGF $\beta$ and stimulating the release of PGJ2, AjA probably promotes PPAR- $\gamma$ signalling in dcSSc fibroblasts.

We also show that oral administration of AjA attenuates markedly the dermal fibrosis induced in DBA/2J mice by bleomycin. AjA reduces fibroblast activation, ECM deposition, and subsequent dermal thickening. Bleomycin-induced dermal fibrosis is considered a reliable experimental model for SSc that reflects the early stages of the disease, with increased deposition of collagen and other ECM components, migration of inflammatory cells into the skin and atrophy of the adipose tissue. ${ }^{30}$ It is still unclear why in SSc, atrophy of the fat tissue occurs, in favour of connective tissue replacement. However, recent data suggest that PPAR- $\gamma$ might play a central role in regulating the balance between adipogenesis and fibrogenesis. ${ }^{19}$ Accumulating evidence suggests that increased activation of PPAR- $\gamma$ favours adipogenesis, whereas decreased PPAR- $\gamma$ favours tissue fibrosis. According to this model, the downregulation of PPAR- $\gamma$ seen in SSc would enhance recruitment of mesenchymal precursors, stimulate release of collagen from resident fibroblasts and inhibit adipogenic differentiation. ${ }^{19}$ In accordance, reduction of PPAR- $\gamma$ in Wnt-10b-transgenic mice suppresses adipogenesis while promoting reciprocal fibrogenesis. ${ }^{31}$ Consistent with this hypothesis, AjA stimulates the differentiation of embryogenic fibroblastic 3T3 L1 cells into adipocytes, a process that is known to be mediated by PPAR- $\boldsymbol{.}^{10}$

Based on these results, we suggest that AjA might exert its antifibrotic effects by restoring the defective PPAR- $\gamma$ activation in dcSSc fibroblasts. We observed that AjA stimulates the expression of PPAR- $\gamma$ in dcSSc fibroblasts, upregulating expression of its endogenous ligand 15d-PGJ2. We showed that activation of PPAR- $\gamma$ is essential for the antifibrotic effects of AjA in vitro and that inhibition of PPAR- $\gamma$ disrupts the inhibitory effects of AjA on collagen synthesis. In addition, treatment of bleomycin-challenged mice with AjA prevents fibrosis and also the characteristic atrophy of the subcutis. 
Results of the studies presented in this paper show that $\mathrm{Aj} A$ reduces infiltration of the dermis by inflammatory cells. Since the mouse model of bleomycin-induced fibrosis is responsive to anti-inflammatory drugs, ${ }^{23}$ we might assume that anti-inflammatory effects of AjA might also contribute to its antifibrotic activity in vivo. We also observed direct antifibrotic effects of $\mathrm{Aj} \mathrm{A}$ in in vivo models largely independent of inflammation-for example, in mice overexpressing a constitutively active TGF $\beta$ receptor I. Moreover, in murine models of established fibrosis, we demonstrated that $\mathrm{Aj} A$ reduces progression of fibrosis. However, treatment with $\mathrm{AjA}$ does not induce regression of preexisting fibrosis in these models.

In conclusion, we demonstrate that AjA exerts potent antifibrotic effects in vivo, which in vitro are mediated by PPAR- $\gamma$ signalling. Cannabinoids and PPAR- $\gamma$ agonists might be ideal drugs to target SSc since they can modulate fibrosis, inflammation and vasodilatation, all of which are dysregulated in SSc. Our findings have direct translational implications, because a proof-of-concept trial with AjA for the treatment of SSc is in preparation.

Acknowledgements The authors are grateful to Professor Robert B Zurier, MD, Professor of Medicine, University of Massachusetts Medical School (Worcester, Massachusetts, USA), for advice and critical reading of the manuscript. The authors thank JB Therapeutics (Massachusetts, USA) which kindly provided ajulemic acid for all the experiments presented in this paper.

Contributors (1) Guarantor of integrity of the entire study: EGG, ES, JHWD; (2) study concept and design: EGG, ES, JHWD; (3) literature research: EGG, ES, PEL, PLC, MG, FLP; (4) in vivo and in vitro experiments: EB, AA, KP, SL, CM, ML, EG; (5) data collection and interpretation: $E G G, E S, E B, C B ;(6)$ statistical analysis: EGG, EB, CB; (7) manuscript editing: EGG, ES, JWHD, MG, PEL, PLC; (8) final approval of manuscript: EGG, ES, JWHD, FLP, MG, PEL, PLC, EB, AA, KP, SL, CB, CM, ML,EG

Competing interests None.

Provenance and peer review Not commissioned; externally peer reviewed.

\section{REFERENCES}

1. Klein TW. Cannabinoid-based drugs as anti-inflammatory therapeutics. Nat Rev Immunol 2005;5:400-11.

2. Pertwee RG. Emerging strategies for exploiting cannabinoid receptor agonists as medicines. Br J Pharmacol 2009;156:397-411.

3. Teixeira-Clerc $\mathbf{F}$, Julien B, Grenard $P$, et al. The endocannabinoid system as a novel target for the treatment of liver fibrosis. Pathol Biol 2008;56:36-8.

4. Michalski CW, Maier M, Erkan M, et al. Cannabinoids reduce markers of inflammation and fibrosis in pancreatic stellate cells. PLoS One 2008;3:e1701.

5. Pertwee RG. Pharmacological actions of cannabinoids. Handb Exp Pharmacol 2005; 168:1-51

6. Akhmetshina A, Dees C, Busch N, et al. The cannabinoid receptor CB2 exerts antifibrotic effects in experimental dermal fibrosis. Arthritis Rheum 2009;60:1129-36.

7. Marquart S, Zerr P, Akhmetshina A, et al. Inactivation of the cannabinoid receptor CB1 prevents leukocyte infiltration and experimental fibrosis. Arthritis Rheum 2010;62:3467-76.

8. Servettaz A, Kavian N, Nicco C, et al. Targeting the cannabinoid pathway limits the development of fibrosis and autoimmunity in a mouse model of systemic sclerosis. Am J Pathol 2010;177:187-96.

9. Balistreri E, Garcia-Gonzalez E, Selvi E, et al. The cannabinoid WIN55, 212-2 abrogates dermal fibrosis in scleroderma bleomycin model. Ann Rheum Dis 2011;70:695-9.
10. Burstein S. Ajulemic acid (IP-751): synthesis, proof of principle, toxicity studies, and clinical trials. AAPS J 2005;7:E143-8.

11. Salim K, Schneider U, Burstein S, et al. Pain measurements and side effect profile of the novel cannabinoid ajulemic acid. Neuropharmacology 2005;48:1164-71.

12. Vann RE, Cook CD, Martin BR, et al. Cannabimimetic properties of ajulemic acid. J Pharmacol Exp Ther 2007;320:678-86.

13. Karst M. Comments on "cannabimimetic properties of ajulemic acid". J Pharmacol Exp Ther 2007;322:420-1.

14. Liu J, Li H, Burstein SH, et al. Activation and binding of peroxisome proliferatoractivated receptor gamma by synthetic cannabinoid ajulemic acid. Mol Pharmacol 2003;63:983-92.

15. Burstein S. PPAR-gamma: a nuclear receptor with affinity for cannabinoids. Life Sci 2005; 77:1674-84

16. Sun Y, Alexander SP, Garle MJ, et al. Cannabinoid activation of PPAR alpha; a nove neuroprotective mechanism. Br J Pharmacol 2007;152:734-43.

17. Giuliano M, Pellerito O, Portanova P, et al. Apoptosis induced in HepG2 cells by the synthetic cannabinoid WIN: involvement of the transcription factor PPARgamma. Biochimie 2009:91:457-65.

18. O'Sullivan SE. Cannabinoids go nuclear: evidence for activation of peroxisome proliferator-activated receptors. Br J Pharmacol 2007;152:576-82.

19. Wei J, Ghosh AK, Sargent JL, et al. PPAR $\gamma$ downregulation by TGFß in fibroblast and impaired expression and function in systemic sclerosis: a novel mechanism for progressive fibrogenesis. PLoS One 2010;5:e13778.

20. Genovese T, Cuzzocrea S, Di Paola R, et al. Effect of rosiglitazone and 15-deoxyDelta12,14-prostaglandin J2 on bleomycin-induced lung injury. Eur Respir J 2005;25:225-34.

21. Kapoor M, McCann M, Liu S, et al. Loss of peroxisome proliferator-activated receptor gamma in mouse fibroblasts results in increased susceptibility to bleomycin-induced skin fibrosis. Arthritis Rheum 2009;60:2822-9.

22. Varga J, Abraham D. Systemic sclerosis: a prototypic multisystem fibrotic disorder. J Clin Invest 2007;117:557-67.

23. Beyer C, Schett G, Distler 0, et al. Animal models of systemic sclerosis: prospects and limitations. Arthritis Rheum 2010;62:2831-44.

24. Akhmetshina A, Venalis P, Dees C, et al. Treatment with imatinib prevents fibrosis in different preclinical models of systemic sclerosis and induces regression of established fibrosis. Arthritis Rheum 2009:60:219-24.

25. Avouac J, Palumbo K, Tomcik M, et al. Inhibition of AP-1 signaling abrogates TGF-ß mediated activation of fibroblasts and prevents experimental fibrosis. Arthritis Rheum 2011.

26. LeRoy EC, Black C, Fleischmajer R, et al. Scleroderma (systemic sclerosis): classification, subsets and pathogenesis. J Rheumatol 1988;15:202-5.

27. Garcia-Gonzalez $\mathbf{E}$, Selvi E, Balistreri E, et al. Cannabinoids inhibit fibrogenesis in diffuse systemic sclerosis fibroblasts. Rheumatology (Oxford) 2009;48:1050-6.

28. Wei J, Bhattacharyya S, Tourtellotte WG, et al. Fibrosis in systemic sclerosis: Emerging concepts and implications for targeted therapy. Autoimmun Rev 2010;21:267-75

29. Kulkarni AA, Thatcher TH, Olsen KC, et al. PPAR- $\gamma$ ligands repress TGF $\beta$-induced myofibroblast differentiation by targeting the PI3K/Akt pathway: implications for therapy of fibrosis. PLoS One 2011;6:e15909.

30. Yamamoto T. Animal model of systemic sclerosis. J Dermatol 2010;37:26-41.

31. Wei J, Melichian D, Komura K, et al. Canonical Wnt signaling induces skin fibrosis and subcutaneous lipoatrophy: a novel mouse model for scleroderma? Arthritis Rheum 2011;63:1707-17.

32. Avouac J, Fürnrohr BG, Tomcik M, et al. Inactivation of the transcription factor STAT4 prevents inflammation-driven fibrosis in systemic sclerosis animal models. Arthritis Rheum 2010;30:800-9.

33. Reich N, Maurer B, Akhmetshina A, et al. The transcription factor Fra-2 regulates the production of extracellular matrix in systemic sclerosis. Arthritis Rheum 2010;62:280-90.

34. Venalis $\mathbf{P}$, Maurer $B$, Akhmetshina A, et al. Lack of inhibitory effects of the antifibrotic drug imatinib on endothelial cell functions in vitro and in vivo. J Cell Mol Med 2009; 13:4185-91. 


\section{ARD}

Synthetic cannabinoid ajulemic acid exerts potent antifibrotic effects in experimental models of systemic sclerosis

Estrella Garcia Gonzalez, Enrico Selvi, Epifania Balistreri, Alfiya Akhmetshina, Katrin Palumbo, Sauro Lorenzini, Pietro Enea Lazzerini, Cinzia Montilli, Pier Leopoldo Capecchi, Monica Lucattelli, Caterina Baldi, Elena Gianchecchi, Mauro Galeazzi, Franco Laghi Pasini and Jörg H W Distler

Ann Rheum Dis 2012 71: 1545-1551 originally published online April 4, 2012

doi: 10.1136/annrheumdis-2011-200314

Updated information and services can be found at:

http://ard.bmj.com/content/71/9/1545

\section{These include:}

\section{Supplementary Supplementary material can be found at:}

Material http://ard.bmj.com/content/suppl/2012/04/04/annrheumdis-2011-2003 14.DC1.html

References This article cites 33 articles, 6 of which you can access for free at: http://ard.bmj.com/content/71/9/1545\#BIBL

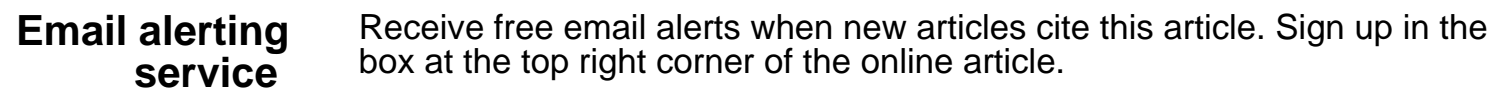

\section{Notes}

To request permissions go to:

http://group.bmj.com/group/rights-licensing/permissions

To order reprints go to:

http://journals.bmj.com/cgi/reprintform

To subscribe to BMJ go to:

http://group.bmj.com/subscribe/ 